\title{
Uncoupling of the profibrotic and hemostatic effects of thrombin in lung fibrosis
}

\author{
Barry S. Shea, ${ }^{1,2}$ Clemens K. Probst, ${ }^{2}$ Patricia L. Brazee, ${ }^{2}$ Nicholas J. Rotile, ${ }^{3}$ Francesco Blasi, ${ }^{3}$ \\ Paul H. Weinreb, ${ }^{5}$ Katharine E. Black, ${ }^{2}$ David E. Sosnovik, ${ }^{3}$ Elizabeth M. Van Cott, ${ }^{4}$ \\ Shelia M. Violette, ${ }^{5}$ Peter Caravan, ${ }^{3}$ and Andrew M. Tager ${ }^{2}$ \\ 'Division of Pulmonary, Critical Care and Sleep Medicine, Alpert Medical School of Brown University and Rhode Island \\ Hospital, Providence, Rhode Island, USA. ${ }^{2}$ Division of Pulmonary and Critical Care Medicine and Center for Immunology \\ and Inflammatory Diseases, ${ }^{3} \mathrm{~A}$. A. Martinos Center for Biomedical Imaging, Department of Radiology, ${ }^{4}$ Department of \\ Pathology, Massachusetts General Hospital and Harvard Medical School, Boston, Massachusetts, USA. \\ ${ }^{5}$ Biogen, Cambridge, Massachusetts, USA.
}

Fibrotic lung disease, most notably idiopathic pulmonary fibrosis (IPF), is thought to result from aberrant wound-healing responses to repetitive lung injury. Increased vascular permeability is a cardinal response to tissue injury, but whether it is mechanistically linked to lung fibrosis is unknown. We previously described a model in which exaggeration of vascular leak after lung injury shifts the outcome of wound-healing responses from normal repair to pathological fibrosis. Here we report that the fibrosis produced in this model is highly dependent on thrombin activity and its downstream signaling pathways. Direct thrombin inhibition with dabigatran significantly inhibited protease-activated receptor- 1 (PAR1) activation, integrin $\alpha_{v} \beta_{6}$ induction, TCF- $\beta$ activation, and the development of pulmonary fibrosis in this vascular leak-dependent model. We used a potentially novel imaging method - ultashort echo time (UTE) lung magnetic resonance imaging (MRI) with the gadolinium-based, fibrin-specific probe EP-2104R - to directly visualize fibrin accumulation in injured mouse lungs, and to correlate the antifibrotic effects of dabigatran with attenuation of fibrin deposition. We found that inhibition of the profibrotic effects of thrombin can be uncoupled from inhibition of hemostasis, as therapeutic anticoagulation with warfarin failed to downregulate the PAR1/ $\alpha_{v} \beta_{6} /$ TCF- $\beta$ axis or significantly protect against fibrosis. These findings have direct and important clinical implications, given recent findings that warfarin treatment is not beneficial in IPF, and the clinical availability of direct thrombin inhibitors that our data suggest could benefit these patients.

Authorship note: C.K. Probst and P.L. Brazee contributed equally to this work.

Conflict of interest: A.M. Tager has received research support from Biogen and Boehringer Ingelheim within the past year. P. Caravan is a stockholder of Collagen Medical LLC, Factor 1A LLC, Reveal Pharmaceuticals, a consultant to Guerbet, UCB Pharma, Collagen Medical $\mathrm{LLC}$, and has received grant/research support from Agilent, Biogen, Pfizer, Siemens.

Submitted: February 24, 2016

Accepted: March 21, 2017

Published: May 4, 2017

\section{Reference information:}

JCI Insight. 2017;2(9):e86608.

https://doi.org/10.1172/jci.

insight.86608.

\section{Introduction}

Responses to tissue injury involve complex cascades of biological processes. When these responses are appropriate in timing, magnitude, and balance, they result in restoration of normal tissue structure and function. Dysregulation of these responses, however, can result in tissue fibrosis and organ failure. The quintessential fibrosing lung disease, idiopathic pulmonary fibrosis (IPF), is thought to develop in just this manner - as a consequence of aberrant or overexuberant wound-healing responses to repetitive, microscopic injury to the lung epithelium $(1,2)$. There have been great advances over the past 2 decades in elucidating the potential causes of lung injury in IPF, the specific wound-healing pathways that become dysregulated after this injury, and the genetic and environmental factors that may promote susceptibility to lung injury and/or profibrotic responses to it (3). As a result of these and other advances, there are now 2 new therapeutic agents that have been shown to slow the rate of disease progression in IPF $(4,5)$.

Injury to the lung epithelium triggers several cardinal wound-healing responses, analogous to those induced by cutaneous injury, including: (a) an inflammatory response, with increased vascular permeability and recruitment and/or activation of cells of the innate and adaptive immune systems; (b) extravascular coagulation and formation of wound clots; (c) the recruitment and proliferation of fibroblasts, which become activated, synthesize collagen and other extracellular matrix components, and contract the wound; and finally (d) re-epithelialization $(6,7)$. Dysregulation of each of these individual processes has been 
described in IPF, but whether dysregulation of all, or only some of these processes, is required to produce pulmonary fibrosis in individual patients has yet to be determined. There has been intense investigation into the potential role of inflammatory cells, including those of both the adaptive and innate immune systems, in IPF pathogenesis (8). In contrast, relatively little attention has focused on the role of the noncellular component of inflammatory responses — increased vascular permeability — in the development of lung fibrosis. There are data suggesting that it may play an important role, however. The presence of increased vascular permeability in the lungs of IPF patients, as measured by clearance of inhaled ${ }^{99 \mathrm{~m}}$ Tc-diethylenetriaminepentacetate ( $\left.{ }^{99 \mathrm{~m}} \mathrm{Tc}-\mathrm{DTPA}\right)$, was described over 25 years ago. Furthermore, the extent of this increased permeability correlated with disease progression $(9,10)$. These observations have since been corroborated by other investigators using similar methodology, or by measuring the lung protein permeability index $(11,12)$.

Although lung vascular permeability is increased in IPF, the potential mechanisms through which it may contribute to IPF pathogenesis have not yet been established. Increased vascular permeability could contribute to IPF pathogenesis by promoting extravascular coagulation. There is a well-known hemostatic imbalance in IPF lungs; specifically, there is increased procoagulant activity and decreased fibrinolytic activity, resulting in increased fibrin deposition $(13,14)$. There are also many investigations that have demonstrated profibrotic effects of coagulation proteinases and antifibrotic effects of fibrinolytic proteinases in the lung (15-18). Although local production of coagulation proteins has been described in the lung following injury (19), increased vascular permeability can promote extravascular coagulation through the extravasation of plasma clotting factors into the injured airspaces, where exposure to tissue factor initiates the coagulation cascade. Fibrin-rich wound clots can serve as scaffolds into which fibroblasts migrate and then proliferate and synthesize mature extracellular matrix in wound healing. However, 2 independent groups found that fibrinogen-null mice were not protected from lung fibrosis in the bleomycin mouse model, indicating that lung fibrosis can develop independently of fibrin $(20,21)$. More recent data have demonstrated that the coagulation proteinases, most notably thrombin but also factor $\mathrm{Xa}$, can promote fibrosis through interaction with specific cell surface receptors, the proteinase-activated receptors (PARs) 1-4, particularly PAR1 (17, 19 , 22-24). Thrombin activates PAR1 by proteolytic cleavage of the N-terminal extracellular domain, revealing a new N-terminus that acts as a tethered ligand for the receptor (25). In epithelial cells, activation of PAR1 results in cytoskeletal rearrangements that cause a conformational change in the epithelium-specific cell surface integrin $\alpha_{v} \beta_{6}$. In response, the $\alpha_{v} \beta_{6}$ integrin, which binds latent TGF- $\beta$ in the extracellular matrix, induces the release of active TGF- $\beta$ from its latency-associated peptide, allowing it to interact with its receptors and exert its profibrotic effects (26). The $\alpha_{v} \beta_{6}$ integrin has been shown to be critical for the development of lung fibrosis in multiple animal models (27-29), and its expression is increased in human fibrotic lung tissue and correlates inversely with survival $(28,30)$. Accordingly, an anti- $\alpha_{\mathrm{v}} \beta_{6}$ antibody is currently being evaluated in a phase II clinical trial in IPF (clinicaltrials.gov identifier: NCT01371305).

We previously described a model in which exaggeration of endothelial barrier disruption and vascular leak after lung injury shift the wound-healing response from one which results in normal repair to one which results in lung fibrosis (31). Specifically, we found that administration of low-dose intratracheal bleomycin to mice $(0.1 \mathrm{U} / \mathrm{kg})$ by itself causes only a mild, self-limited lung injury, without the development of lung fibrosis. However, when the endothelial barrier-disrupting agents FTY720 (a nonselective sphingosine 1-phosphate [S1P]-receptor modulator) or AUY954 (an S1P-receptor 1 [S1P $]$-selective modulator) are administered in addition to this low-dose bleomycin challenge, there is an increase in lung vascular leak and accompanying intra-alveolar coagulation, and ultimately the development of extensive lung fibrosis (31). We propose that in this vascular leak-dependent model of lung fibrosis, increased vascular permeability promotes the development of lung fibrosis by augmenting activation of the coagulation cascade in the injured airspaces, thereby activating the profibrotic thrombin/PAR $1 / \alpha_{\mathrm{v}} \beta_{6} / \mathrm{TGF}-\beta$ axis. Here we demonstrate that, consistent with this hypothesis, the development of lung fibrosis in this model is entirely dependent on thrombin activity and $\alpha_{v} \beta_{6}$. It is uncoupled from hemostasis and lung fibrin deposition, however, as we show that therapeutic anticoagulation with warfarin is unable to significantly protect against lung fibrosis in this model. This dissociation of the role of thrombin activity from hemostasis has important and immediate clinical implications for individuals with IPF, given recent findings that warfarin treatment is not beneficial in IPF (32), and the clinical availability of direct thrombin inhibitors that our data suggest could benefit these patients. In addition, these findings may have widespread relevance for pathological fibrosis, as the thrombin/PAR $1 / \alpha_{v} \beta_{6} /$ TGF- $\beta$ axis has also been implicated in the development of fibrosis in other organ systems, including the liver and kidneys (33-38). 

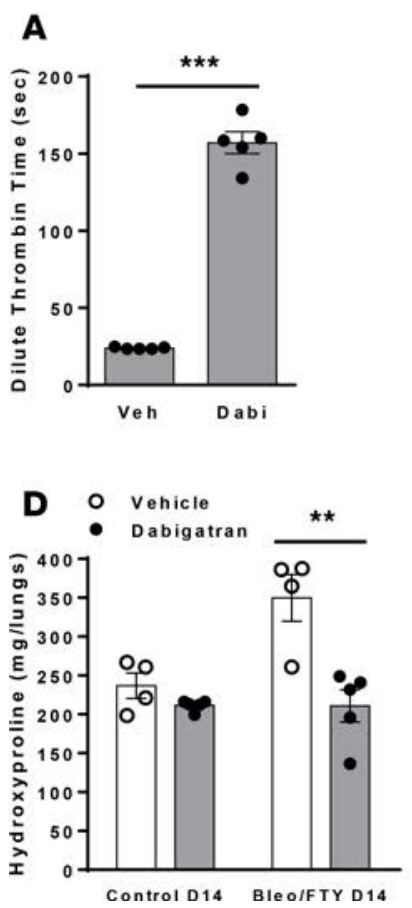
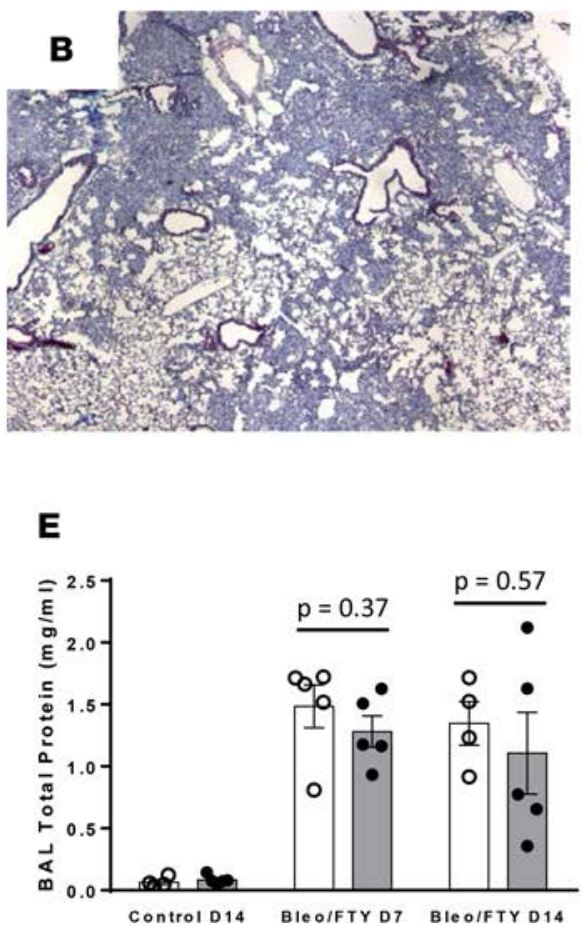

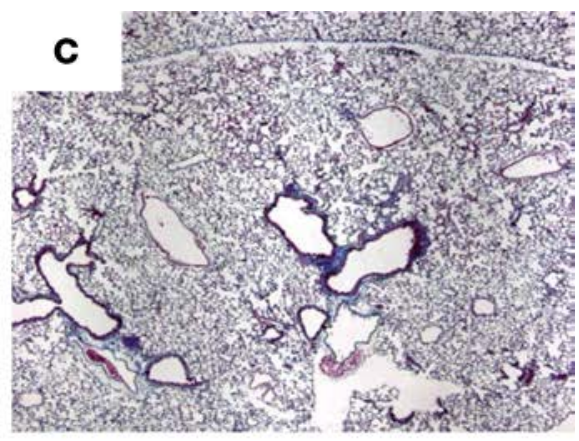

$\mathbf{F}$

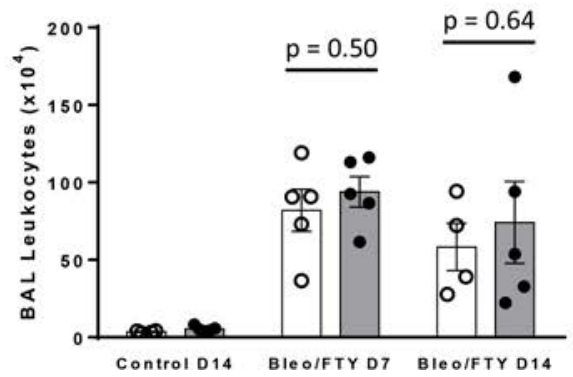

Figure 1. Thrombin inhibition attenuates fibrosis in a vascular leak-dependent model. (A) Plasma dilute thrombin times in mice treated with dabigatran (Dabi) or vehicle (Veh) for 1 week (representative of 2 independent experiments). (B and C) Representative images of Masson's trichromestained mouse lung sections at day 14 after bleomycin + FTY720 challenge with vehicle (B) or dabigatran (C) treatment ( $n=3 /$ group). Original magnification, $\times 40$. (D-F) Measurement of total lung hydroxyproline content (D), bronchoalveolar lavage (BAL) total protein concentration (E), and BAL total leukocytes (F) at day 7 (D7) and/or D14 in mice challenged with intratracheal (i.t.) PBS + i.p. sterile water (control) or i.t. bleomycin + i.p. FTY720 (Bleo/FTY) and treated with dabigatran or vehicle (hydroxyproline data are representative of 11 independent experiments). Individual data points are presented, along with as mean $\pm \mathrm{SEM}$. ${ }^{* *} P=0.006,{ }^{* *} P<0.0001$ by 2 -tailed $t$ tests.

\section{Results}

Thrombin inhibition attenuates lung fibrosis in a vascular leak-dependent model. We assessed the ability of the direct thrombin inhibitor dabigatran to attenuate the development of lung fibrosis in a vascular leak-dependent model, produced by the administration of low-dose intratracheal bleomycin on day 0 and intraperitoneal FTY720 three times per week, as previously described (31). Mice were fed with dabigatran-containing or control chow starting at day 0 . Plasma dilute thrombin times (dTTs) measured after 1 week confirmed that this method of dabigatran administration achieved systemic anticoagulation (Figure 1A), with dTT values within the range seen in patients treated with therapeutic doses of dabigatran $(39,40)$. As previously shown (31), the combination of low-dose bleomycin and FTY720 resulted in extensive lung collagen deposition, as assessed qualitatively with Masson's trichrome-stained lung sections from mice sacrificed at day 14 (Figure 1B). Quantitative assessment of total lung collagen by measurement of lung hydroxyproline content confirmed a significant increase in lung fibrosis at day 14 after bleomycin + FTY720 challenge (Figure 1D). Dabigatran treatment completely blocked the deposition of collagen (Figure 1C) and the increase in lung hydroxyproline content (Figure 1D), indicating that the development of lung fibrosis in this model is highly dependent on thrombin.

It has previously been shown that thrombin inhibition, or loss of the major thrombin receptor, PAR1, significantly attenuates the development of lung injury and inflammation after standard, high-dose bleomycin challenge in mice $(17,22,23)$. To determine whether thrombin inhibition similarly attenuated lung injury in our vascular leak-dependent model, we measured bronchoalveolar lavage (BAL) total protein content (Figure 1E), total leukocyte counts (Figure 1F), and differential leukocyte counts (data not shown) at days 7 and 14 after low-dose bleomycin + FTY720 with and without dabigatran treatment. Dabigatran did not appear to protect against lung injury by these measures, as we did not detect significant reductions in BAL total protein content or inflammatory cell recruitment at either of these time points. To more specifically assess vascular leak at day 7, we also measured the BAL concentrations of the plasma macromolecules albumin and $\alpha-2$ macroglobulin at day 7 (Supplemental Figure 1; supplemental material available 
A
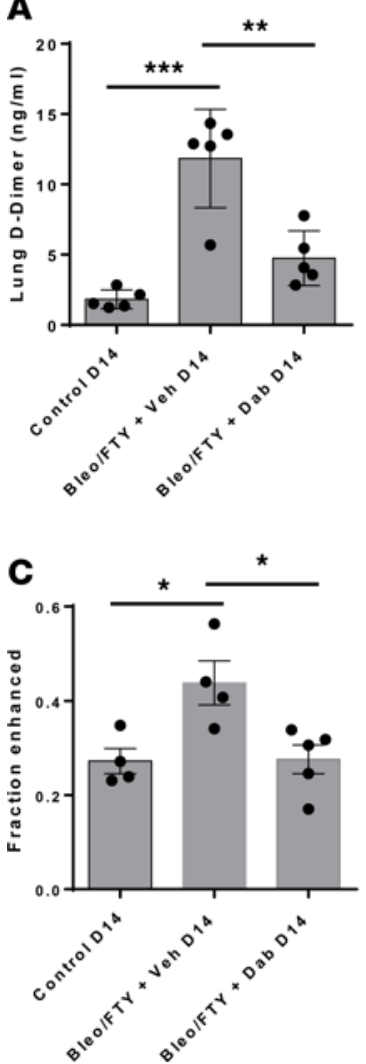

B

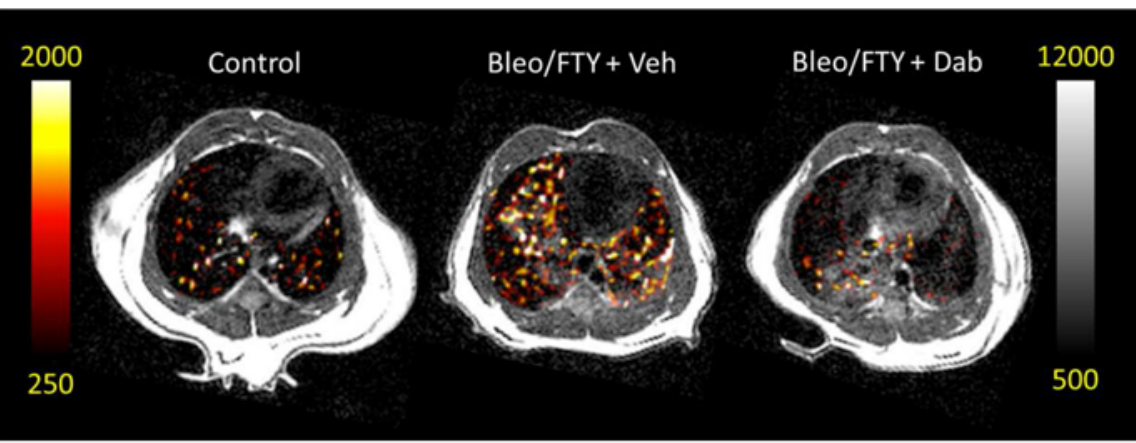

D

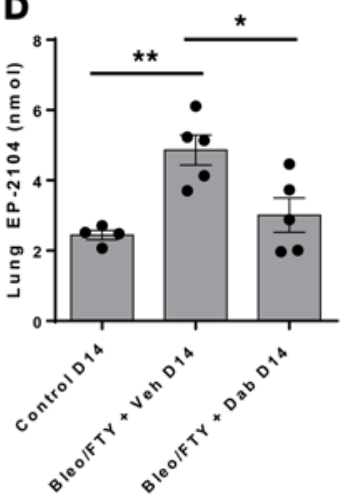

E

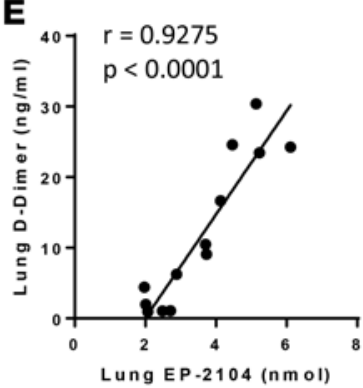

F

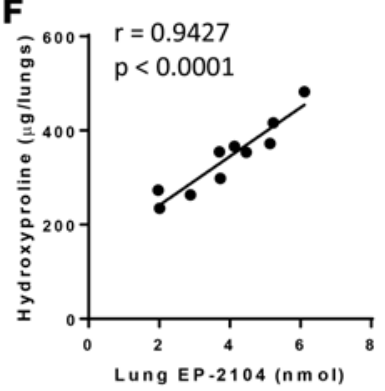

Figure 2. Thrombin inhibition attenuates lung fibrin deposition. (A) Extravascular lung D-dimer content at day 14 in control mice and mice challenged with bleomycin + FTY720 and treated with vehicle (Veh) or dabigatran (Dab). (B-F) Data from ultrashort echo time (UTE) lung MRI with the gadoliniumbased, fibrin-specific probe EP-2104R at day 14 in control mice and mice challenged with bleomycin + FTY720 and treated with vehicle or dabigatran ( $n=$ 4-5/group). (B) Representative images from EP-2104R-enhanced lung MRI. (C) Semiquantitative analysis of the imaging data from EP-2104R-enhanced UTE lung MRI. (D) Quantitative assessment of lung fibrin deposition by measurement of total lung EP-2104R content. (E) Correlation between total lung D-dimer content and total lung EP-2104R content of mice challenged with bleomycin + FTY720 and treated with dabigatran versus vehicle. (F) Correlation between total lung hydroxyproline content and total lung EP-2104R content in mice challenged with bleomycin + FTY720 and treated with dabigatran versus vehicle. Data are representative of 2 independent experiments. Individual data points are presented in graphs, along with mean \pm SEM where applicable. ${ }^{*} P<0.05,{ }^{* *} P<0.005,{ }^{* *} P<0.0005$ by 2 -tailed $t$ tests for indicated pairwise comparisons.

online with this article; https://doi.org/10.1172/jci.insight.86608DS1). Despite the fact that dabigatran treatment did not appear to affect BAL total protein concentration, it did appear to limit the extravasation of albumin and $\alpha-2$ macroglobulin from the plasma into the airspaces, suggesting that dabigatran attenuated to some extent the disruption of the alveolar-capillary barrier in this model.

Thrombin inhibition attenuates lung fibrin deposition. As would be expected, thrombin inhibition caused a decrease in lung extravascular fibrin deposition in this vascular leak-dependent model. As shown in Figure 2A, whole-lung extravascular D-dimer concentration was significantly increased in mice challenged with low-dose bleomycin + FTY720, and this increase was greatly diminished with dabigatran treatment. D-dimer is a marker of fibrin turnover, however, and not a direct assessment of lung fibrin content. Efforts to directly assess tissue fibrin in mice have been complicated by the lack of antibodies able to distinguish between mouse fibrin and its precursor, fibrinogen, and by the insoluble nature of deposited fibrin, making it difficult to extract for common quantitative analyses, e.g., Western blotting. To directly assess the extent of lung fibrin deposition in this model, we used ultrashort echo time magnetic resonance imaging (UTE-MRI) of the lungs in combination with a gadolinium (Gd)-based, fibrin-binding molecular probe EP-2104R. UTE-MRI is a technique for lung imaging that overcomes the numerous air-tissue interfaces which lead to rapid signal decay in conventional proton MRI of the lungs (41-43). EP-2104R has been demonstrated to have high specificity for fibrin compared with fibrinogen, and it has been shown to be a sensitive method for detecting intravascular thrombi with MRI (44-49). As shown in Figure 2, B and C, mice challenged with low-dose bleomycin + FTY720 demonstrated significantly increased lung fibrin 

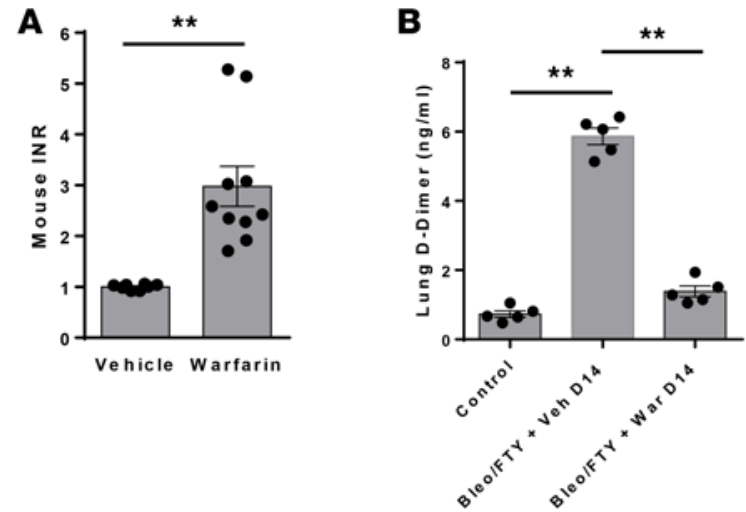

\section{$\mathbf{E}$}

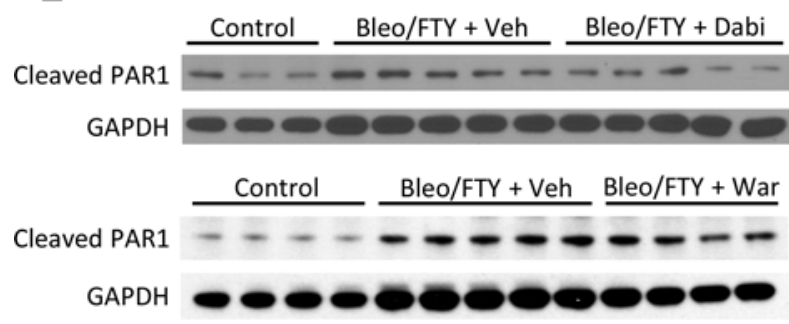

C

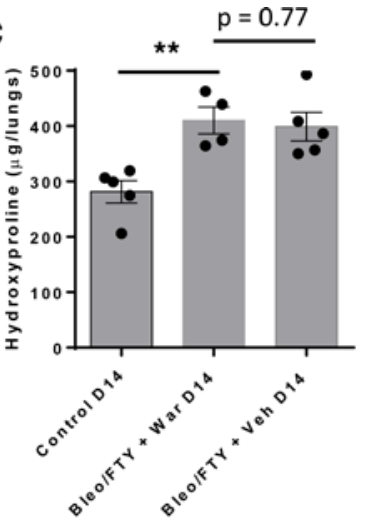

F

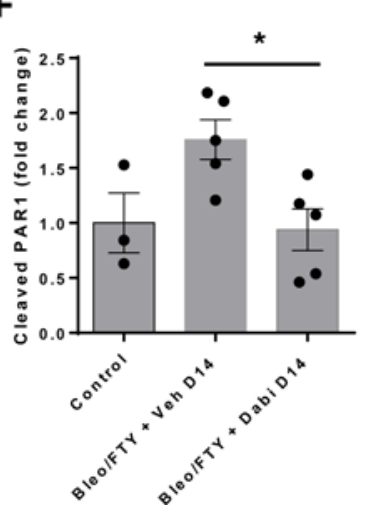

D

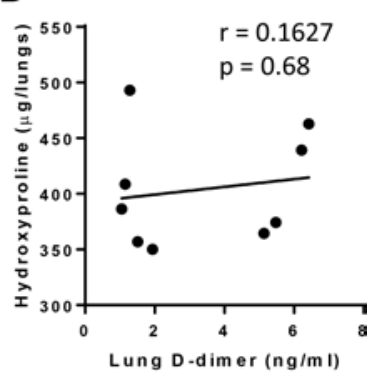

G

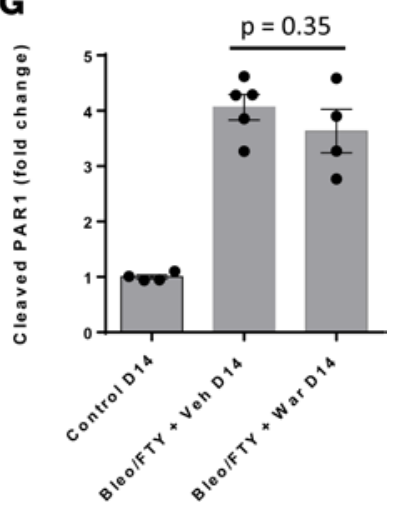

Figure 3. Therapeutic anticoagulation with warfarin does not protect against fibrosis. (A) Mouse plasma international normalized ratios (INRs) after treatment with warfarin or vehicle for 2 weeks. (B and C) Measurement of lung extravascular D-dimer content (B) and total lung hydroxyproline content (C) at day 14 in control mice and mice challenged with bleomycin + FTY720 and treated with vehicle (Veh) or warfarin (War) (hydroxyproline data are representative of 11 independent experiments). (D) Correlation between total lung hydroxyproline content and total lung D-dimer content in mice challenged with bleomycin + FTY720 and treated with warfarin versus vehicle. (E-G) Western blot analyses for cleaved (activated) PAR1 in whole-lung homogenates at day 14 in control mice and mice challenged with bleomycin + FTY720 and treated with dabigatran (Dabi) versus vehicle (E, top panel; densitometry in F) or warfarin versus vehicle (E, bottom panel; densitometry in $\mathbf{G}$ ). Western blot bands for cleaved PAR1 and GAPDH were seen at the expected molecular weights of $\sim 46 \mathrm{kDa}$ and $\sim 37 \mathrm{kDa}$, respectively. Western blot data are representative of at least 2 independent experiments for each analysis. Individual data points are presented in graphs, along with mean \pm SEM where applicable. ${ }^{*} P<0.05,{ }^{* *} P<0.005$ by 2 -tailed $t$ tests for indicated pairwise comparisons.

deposition by EP-2104R-enhanced UTE-MRI, and this increase was almost completely eliminated with dabigatran treatment. Quantification of total EP-2104R in the lungs by biochemical measurement of lung homogenate Gd content confirmed these findings (Figure 2D). In mice challenged with bleomycin + FTY720 there was a strong correlation between lung tissue EP-2104R and D-dimer concentrations (Figure $2 \mathrm{E}$ ), suggesting that lung $\mathrm{D}$-dimer is a useful marker of fibrin deposition in this model. Furthermore, there was also a strong correlation between total lung EP-2104 and the extent of lung fibrosis in these mice as measured by lung hydroxyproline content (Figure $2 \mathrm{~F}$ ), indicating that the extent of protection against lung fibrin deposition as a consequence of thrombin inhibition correlated strongly with protection from fibrosis.

Therapeutic anticoagulation with warfarin does not protect against lung fibrosis. While the data presented above show a correlation between attenuation of lung fibrin deposition after injury and protection against lung fibrosis with thrombin inhibition, a recent placebo-controlled trial of a different anticoagulant, the vitamin $\mathrm{K}$ antagonist warfarin, in patients with IPF failed to show a beneficial effect, and was terminated early due to increased mortality in those subjects in the warfarin arm (32). We consequently hypothesized that the antifibrotic effect of dabigatran in our fibrosis model was due to the inhibition of profibrotic thrombin signaling through PAR1, rather than dabigatran's effects on coagulation and hemostasis per se. To test this hypothesis, we administered warfarin to mice in our fibrosis model at a dose that resulted in therapeutic systemic anticoagulation (Figure 3A). Mice were started on warfarin or vehicle on day -7 , and then challenged with the low-dose bleomycin + FTY720 model as above starting on day 0. Systemic anticoagulation with warfarin had an effect similar to that of dabigatran on extravascular coagulation in the lung, as 

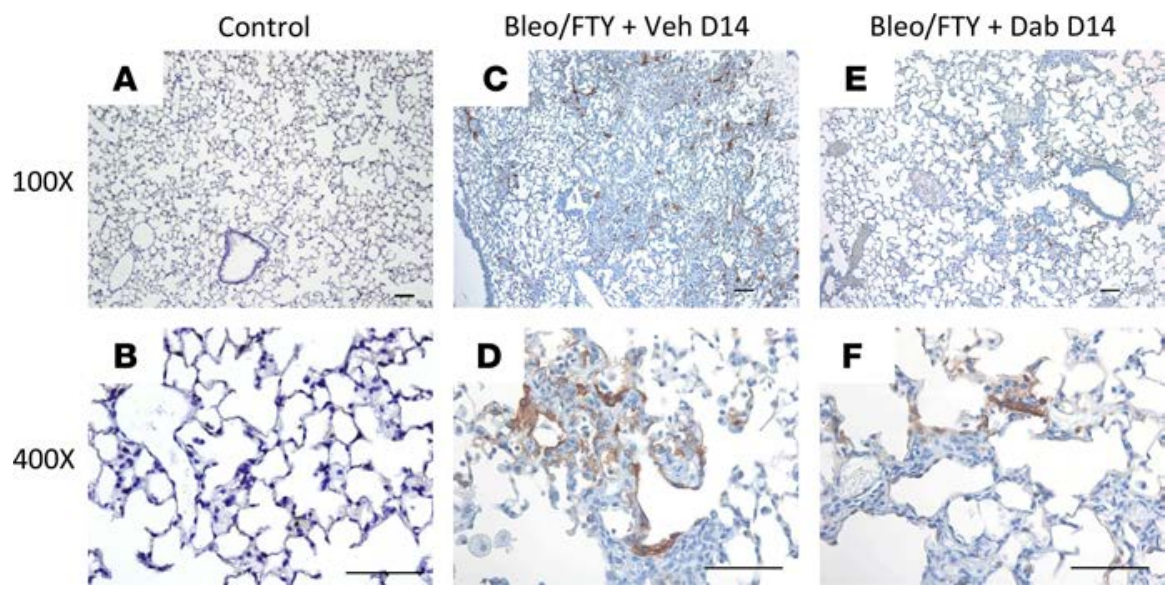

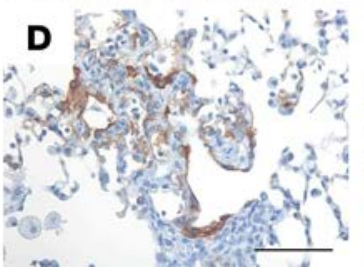

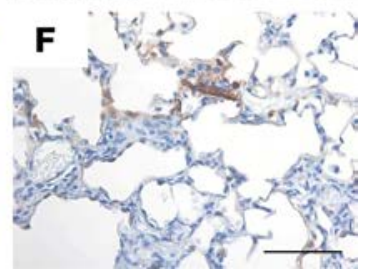

Figure 4. Expression of $\alpha_{v} \beta_{6}$ integrin in a vascular leak-dependent model of lung fibrosis. (A-F) Representative images of immunohistochemical staining for the $\alpha_{v} \beta_{6}$ integrin in day 14 lung sections from control mice (A and $\mathbf{B}$ ) and mice challenged with bleomycin + FTY720 and treated with vehicle (Veh) (C and D) or dabigatran (Dab) (E and F). $n=3 /$ group. Scale bars: $50 \mu \mathrm{m}$.

indicated by almost complete attenuation of the increase in lung D-dimer content (Figure 3B). However, in these same mice in which lung extravascular coagulation was significantly attenuated with warfarin treatment, there was no appreciable reduction in lung fibrosis, as determined by total lung hydroxyproline content (Figure 3C). To increase our power to detect a statistically significant reduction in lung fibrosis with warfarin treatment (and therefore reduced the likelihood of a type II error) we performed a pooled analysis of the day 14 total lung hydroxyproline content from 11 independent experiments comparing warfarin treatment to vehicle treatment in this model. Despite the resulting large sample sizes $(n=53-54$ mice/ group; $89 \%$ power to detect an absolute reduction in total lung hydroxyproline content of $50 \mu \mathrm{g} / \mathrm{lungs}$, with $P<0.05$ ), we failed to identify a statistically significant reduction in lung fibrosis in warfarin-treated mice compared with control mice (Supplemental Figure 2). Consistent with the differing effects of warfarin on lung fibrin deposition and fibrosis, there was no correlation between lung D-dimer and hydroxyproline contents in bleomycin + FTY720-challenged mice treated with warfarin or vehicle (Figure 3D). These data, demonstrating an uncoupling of lung fibrin deposition and lung fibrosis with warfarin treatment, suggest that the antifibrotic effects of dabigatran are specific to inhibition of thrombin itself, rather than to less specific effects on coagulation and hemostasis.

Differential effects of dabigatran and warfarin on thrombin activity. Thrombin signals through the PAR1 receptor in a novel manner dependent on its catalytically active site. Thrombin cleaves the N-terminus of PAR1 between the arginine and serine amino acid residues at positions 41 and 42, creating a new N-terminus that acts as a tethered ligand that binds to and activates the receptor (25). The presence of cleaved PAR1 therefore reflects not only thrombin activation, but also its signaling through this receptor. To specifically investigate the differential effects of thrombin inhibition and vitamin $\mathrm{K}$ antagonism on thrombin/ PAR1 signaling, we assessed the effects of these 2 treatments on the degree of proteolytic cleavage of PAR1 induced in our fibrosis model in vivo by Western blotting with an antibody that recognizes only the cleaved (activated) form of the receptor, anti-cleaved PAR1 (cPAR1; cleaved-Ser ${ }^{42}$ ). We confirmed that this antibody is specific for cleaved rather than native PAR1, as its epitope was only detectable in cells treated with thrombin, and production of this epitope was blocked by inhibition of thrombin's proteolytic activity with dabigatran (Supplemental Figure 3). In vivo, we found whole-lung levels of cleaved PAR1 to be increased at day 14 after bleomycin + FTY720 challenge (Figure 3, E and F). This increase in cleaved PAR1 was completely abrogated in mice treated with dabigatran (Figure 3, E and F). In contrast, therapeutic anticoagulation with warfarin had no significant effect on levels of cleaved PAR1 (Figure 3, E and G), indicating that profibrotic thrombin signaling is not significantly inhibited by vitamin $\mathrm{K}$ antagonism.

Thrombin inhibition attenuates $\alpha_{1} \beta_{6}$ expression and activation of TGF- $\beta$. Prior investigations have suggested that thrombin signaling through PAR1 promotes lung fibrosis by inducing the activation of TGF- $\beta$, in a manner dependent on the epithelial cell surface integrin $\alpha_{\mathrm{v}} \beta_{6}(26,27)$. In the lung, expression of $\alpha_{\mathrm{v}} \beta_{6}$ is restricted to epithelial cells and is upregulated at sites of injury. In its quiescent state, $\alpha_{\mathrm{v}} \beta_{6}$ binds to latent TGF- $\beta$ present in the extracellular matrix. When activated by cytoskeletal rearrangements induced by thrombin/PAR1 signaling, $\alpha_{\mathrm{v}} \beta_{6}$ undergoes a conformational change, releasing active TGF- $\beta$ from its small latent complex and allowing it to interact with its cell surface receptors (50). In addition to activating the $\alpha_{v} \beta_{6}$ integrin, thrombin has also been shown to increase expression of this integrin (35). We assessed the 
A

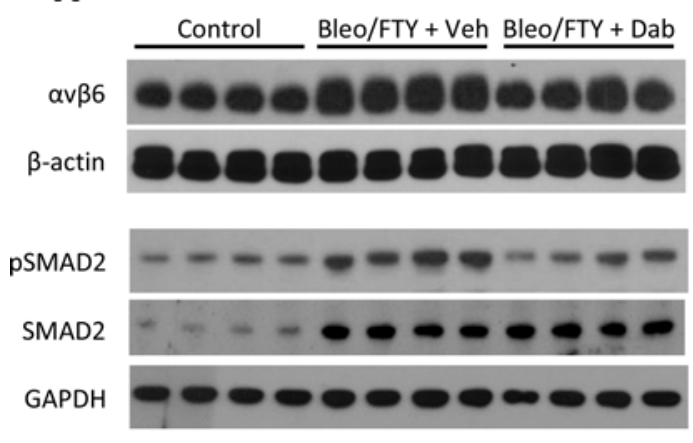

D

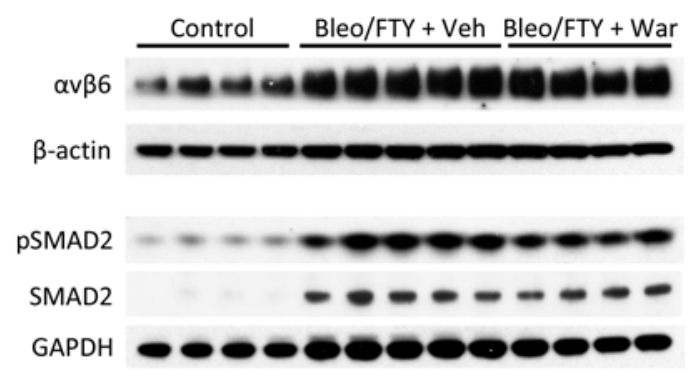

$\mathbf{B}$

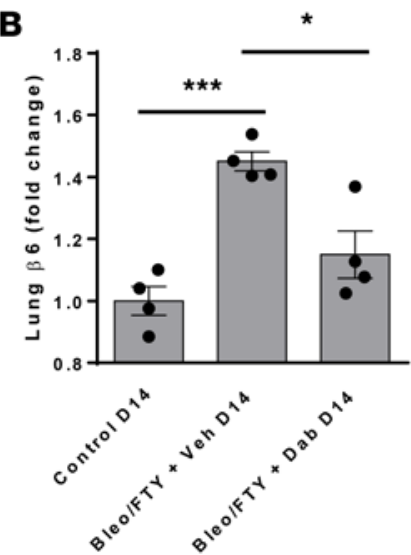

E

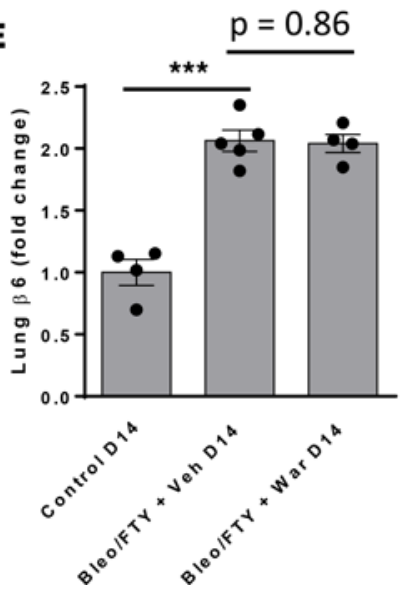

$\mathbf{C}$

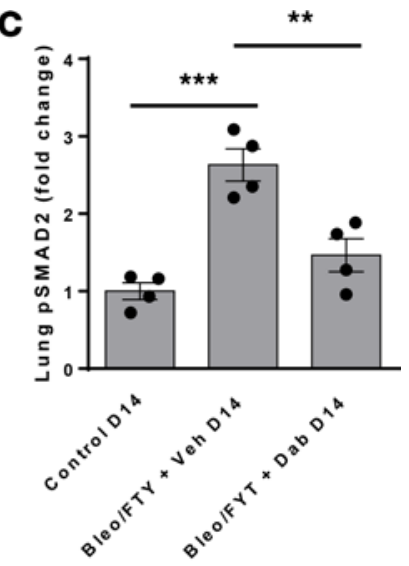

$\mathbf{F}$

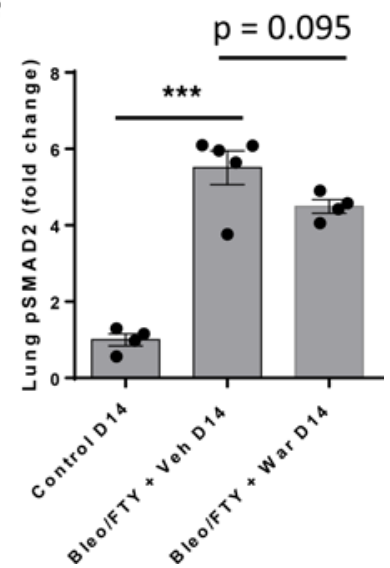

Figure 5. Effects of thrombin inhibition and vitamin $\mathrm{K}$ antagonism on lung $\boldsymbol{\alpha}_{\mathbf{v}} \boldsymbol{\beta}_{6}$ expression and TGF- $\boldsymbol{\beta}$ signaling. (A-C) Western blot analyses for $\alpha \beta$ (A, top panel; densitometry in B), SMAD2 and phosphorylated SMAD2 (p-SMAD2) (A, bottom panel; densitometry in $\mathbf{C}$ ) in whole-lung homogenates at d'ay 14 in control mice and mice challenged with bleomycin + FTY720 and treated with dabigatran versus vehicle. (D-F) Western blot analyses for $\alpha \beta$ (D, top panel; densitometry in E), SMAD2 and p-SMAD2 (D, bottom panel; densitometry in F) in whole-lung homogenates at day 14 in control mice and mice challenged with bleomycin + FTY720 and treated with warfarin versus vehicle. Western blot bands for $\alpha_{v} \beta_{6}$, SMAD2, and p-SMAD2 were seen at the expected molecular weights of $\sim 80 \mathrm{kDa}, \sim 60 \mathrm{kDa}$, and $\sim 60 \mathrm{kDa}$, respectively; bands for $\beta$-actin and GAPDH were seen at the expected molecular weights of $\sim 45 \mathrm{kDa}$ and $\sim 37 \mathrm{kDa}$, respectively. Data are representative of at least 2 independent experiments for each analysis. Individual data points presented in graphs, along with mean \pm SEM where applicable. ${ }^{*} P=0.01,{ }^{* *} P=0.008,{ }^{* * *} P<0.0005$ by 2 -tailed $t$ tests for indicated pairwise comparisons.

expression of $\alpha_{v} \beta_{6}$ in the bleomycin + FTY720 model, and the effect of thrombin inhibition on its expression. As shown in Figure 4, A-D, immunohistochemical staining showed an increase in $\alpha_{\mathrm{v}} \beta_{6}$ expression in this fibrosis model. Consistent with prior literature, this expression appeared to be primarily restricted to sites of injury and localized to the lung epithelium. Treatment with dabigatran significantly attenuated lung $\alpha_{\mathrm{v}} \beta_{6}$ expression, qualitatively by immunohistochemistry (Figure 4, E and F), and quantitatively by Western blotting of whole-lung homogenates (Figure 5, A and B). More relevant than $\alpha_{v} \beta_{6}$ expression, however, is its activation status, as it is the activation of $\alpha_{v} \beta_{6}$ that results in the release of active TGF- $\beta$. Unfortunately, there are no available methods for directly assessing the activation status of $\alpha_{v} \beta_{6}$ in vivo. As a surrogate, we assessed for the presence of active TGF- $\beta$ by measuring the extent of phosphorylated SMAD2 (p-SMAD2) in whole-lung lysates. As shown in Figure 5, the increase in whole-lung p-SMAD2 at day 14 was significantly attenuated with dabigatran treatment (Figure 5, A and C), indicating a decrease in active TGF- $\beta$ levels and consistent with a decrease in $\alpha_{\mathrm{v}} \beta_{6}$ activation. In contrast, treatment with warfarin failed to show a statistically significant decrease in lung $\alpha_{\mathrm{v}} \beta_{6}$ expression at day 14 (Figure 5, D and E). Warfarin treatment also did not significantly reduce lung levels of p-SMAD2 (Figure 5, D and F), indicating that it had no appreciable effect on the presence of active TGF- $\beta$, consistent with an inability to adequately inhibit $\alpha_{\mathrm{v}} \beta_{6}$ mediated TGF- $\beta$ activation.

Antibody blockade of $\alpha_{1} \beta_{6}$ protects against lung fibrosis in a vascular leak-dependent model. Our contrasting findings with thrombin inhibition and vitamin $\mathrm{K}$ antagonism suggest a central role for $\alpha_{\mathrm{v}} \beta_{6}$-mediated activation 
A

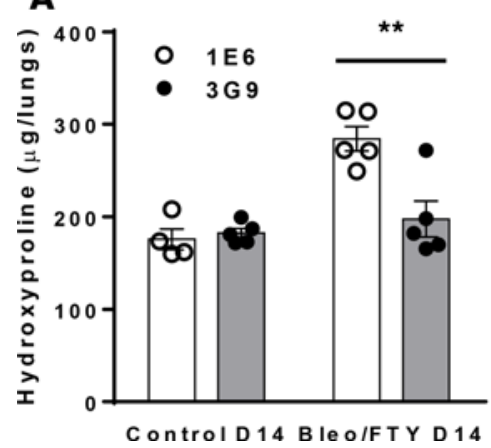

B

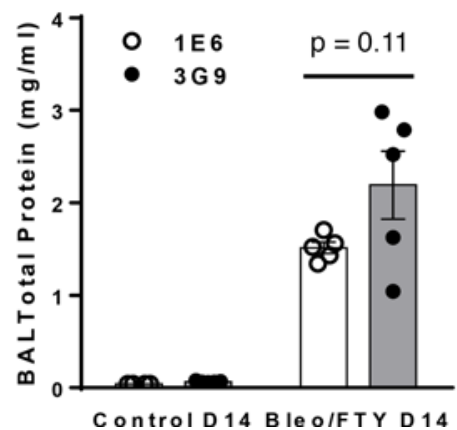

C

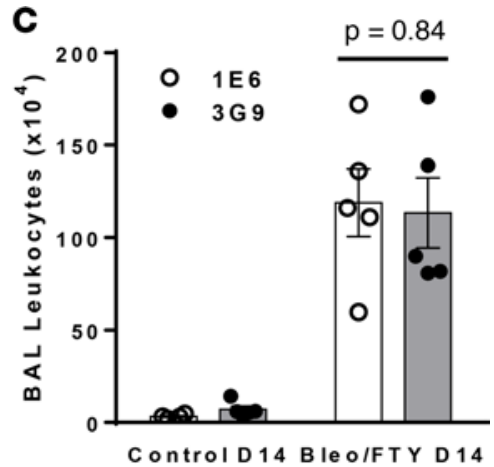

Figure 6. Antibody blockade of $\alpha_{\mathrm{v}} \boldsymbol{\beta}_{6}$ protects against lung fibrosis in a vascular leak-dependent model. (A-C) Measurement of total lung hydroxyproline content (A), bronchoalveolar lavage (BAL) total protein concentration (B), and BAL total leukocytes (C) at day 14 (D14) in mice challenged with intratracheal (i.t.) PBS + i.p. sterile water (control) or i.t. bleomycin + i.p. FTY720 (Bleo/FTY) and treated with the $\alpha_{v} \beta_{6}$-blocking antibody (3G9) versus isotype control antibody (1E6) at $1 \mathrm{mg} / \mathrm{kg}$ s.c. 3 times per week. Data are representative of 4 independent experiments. Individual data points are presented, along with mean $\pm \mathrm{SEM}$. ${ }^{* *} P=0.006$ by 2 -tailed $t$ test.

of TGF- $\beta$ in the development of fibrosis in this model. To confirm the importance of this integrin, we challenged mice with bleomycin + FTY720 and treated them with an anti- $\alpha_{v} \beta_{6}$ blocking antibody (3G9) or control antibody (1E6). As shown in Figure $6 \mathrm{~A}$, anti- $\alpha_{\mathrm{v}} \beta_{6}$ treatment completely inhibited the development of fibrosis at day 14 in this model. Similar to our findings with thrombin inhibition, anti- $\alpha_{v} \beta_{6}$ treatment did not attenuate the increase in BAL total protein or total leukocyte concentrations (Figure 6, B and C), suggesting that its antifibrotic effect is downstream from the development of lung injury in this model.

\section{Discussion}

Aberrant or dysregulated wound-healing responses are thought to play a fundamental role in the development of tissue fibrosis in many organ systems, including the lungs, skin, heart, liver, and kidneys (51). In the lungs, the quintessential fibrosing lung disease of IPF is thought to develop as a consequence of such aberrant wound healing processes in response to repetitive, microscopic lung injury (3). As one of the cardinal responses to tissue injury, increased vascular permeability may therefore play a role in fibrogenesis. The presence of increased vascular permeability in the lungs of IPF patients, and its correlation with disease progression, has been demonstrated in multiple investigations (9-12). A potential mechanistic link between increased vascular permeability and pulmonary fibrosis has not been as well studied, however, and was the focus of this investigation. One consequence of increased vascular permeability is the extravasation of plasma constituents into the surrounding tissue. This loss of compartmentalization between the vascular and extravascular spaces could contribute to fibrosis, as some plasma proteins can activate profibrotic processes when exposed to the environment of injured tissue.

We have previously shown that with prolonged exposure, $\mathrm{S}_{1} \mathrm{P}_{1}$ modulators disrupt endothelial barrier function and increase vascular permeability in vivo (31). In the setting of a low-dose challenge with intratracheal bleomycin in mice - a challenge that by itself causes only mild, transient lung injury and no appreciable fibrosis - this increased vascular permeability is accompanied by the development of significant lung fibrosis (31). We hypothesized that vascular leak contributes to the establishment of lung fibrosis by promoting extravascular coagulation. The increased vascular leak in this model of low-dose bleomycin challenge and $\mathrm{S}_{1} \mathrm{P}_{1}$ inhibition augments extravascular coagulation, giving us a unique opportunity to investigate the antifibrotic potential of therapeutic interventions targeting these processes. Consistent with our hypothesis, here we found that the direct thrombin inhibitor dabigatran completely attenuated the development of fibrosis in this model. While dabigatran treatment predictably attenuated fibrin deposition in the lungs, this decrease in extravascular fibrin did not appear to be responsible for its antifibrotic effects, as similar decreases in lung extravascular coagulation induced by vitamin $\mathrm{K}$ antagonism with warfarin had no effect on the development of lung fibrosis.

Our findings have direct clinical implications for the treatment of patients with IPF. Based on the abundance of data that have suggested an important role for the coagulation cascade in the pathogenesis of fibrotic lung disease, a recent phase III randomized, placebo-controlled clinical trial was undertaken to evaluate the safety and efficacy of anticoagulation with warfarin in IPF (32). Unfortunately, this study was 


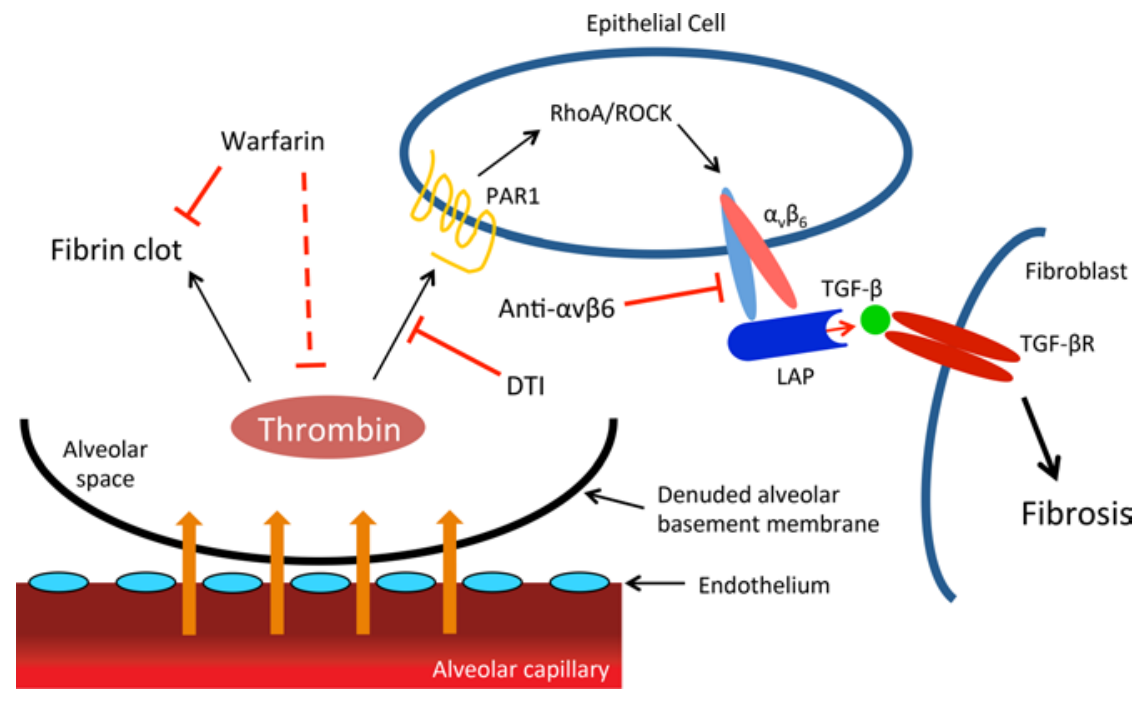

Figure 7. Schematic of the proposed mechanisms linking vascular leak, intra-alveolar thrombin, $\alpha_{v} \beta_{6}$, and TGF- $\beta$ signaling with the development of injury-induced lung fibrosis. As a consequence of lung injury, there is damage to the alveolar epithelium, denudement of the basement membrane, and increased vascular permeability, with extravasation of plasma constituents into the injured alveoli (orange arrows). Among these extravasated plasma contents are the clotting factors, leading to intra-alveolar activation of the coagulation cascade and the generation of active thrombin. In addition to its proteolytic cleavage of fibrinogen to generate fibrin in the airspaces, thrombin also cleaves and activates proteinase activated receptor 1 (PAR1). Based on previous work by Jenkins et al. (ref. 26) and Munger et al. (ref. 27), it has been shown that activation of PAR1 on alveolar epithelial cells consequently activates the $\alpha_{\mathrm{v}} \beta_{6}$ integrin (in a manner dependent on RhoA and Rho kinase, a.k.a. ROCK), which results in the release of extracellular active TCF- $\beta$ from the latency-associated peptide (LAP). In an autocrine fashion, active TGF- $\beta$ can then signal through its receptors (TCF- $\beta R$; here shown on a neighboring fibroblast) to exert its profibrotic effects. Dabigatran, a direct thrombin inhibitor (DTI), or blockade of the $\alpha_{v} \beta_{6}$ integrin interrupts this thrombin/PAR $1 / \alpha_{v} \beta_{6} /$ TGF- $\beta$ axis, thereby halting the progression from lung injury to fibrosis. Conversely, while warfarin is effective at reducing intra-alveolar fibrin accumulation (solid red line), it appears to have only weak effects on overall thrombin activity (dashed red line) in the injured airspaces, which may explain its inability to significantly attenuate the development of fibrosis after lung injury. terminated early, as warfarin treatment not only failed to show a benefit but was also associated with increased mortality. Although the results of this clinical trial would seem to contradict the hypothesis that coagulation contributes to pulmonary fibrosis, many have since pointed out that the coagulation cascade proteinases have important activities beyond their roles in promoting hemostasis (32, 52-54). Most notably thrombin, the terminal coagulation proteinase which cleaves fibrinogen to form fibrin, has well-documented signaling activities that can drive downstream proinflammatory and profibrotic pathways $(17,22,23,26)$. Much of the animal data that supported targeting coagulation in lung fibrosis were generated using approaches that directly interrupt thrombin signaling. Specifically, pharmacologic inhibition of thrombin has been shown to protect against the development of lung fibrosis in mice (17, 23), as has inhibition or genetic deficiency of PAR1 $(22,24)$. It is reasonable to hypothesize that these results were attributable to inhibition of thrombin's profibrotic signaling rather than its fibrin-producing activity. In further support of this hypothesis, 2 independent groups have published findings that mice unable to form fibrin due to genetic deficiency of fibrinogen are not protected from the development of lung fibrosis $(20,21)$. Consequently, although inhibiting hemostasis with warfarin failed to show a benefit in IPF, we hypothesize that direct thrombin inhibition may be helpful for these patients. Our findings demonstrating a clear difference between the effects of thrombin inhibition and vitamin $\mathrm{K}$ antagonism on lung fibrosis, with significant protection from the former but not the latter therapy, support our hypothesis.

PAR1 is the major thrombin receptor, although it can be activated by other coagulation proteinases as well, such as activated factor Xa $(19,55)$. Once activated, PAR1 has been shown to induce intracellular cytoskeletal rearrangements (26). In epithelial cells, these cytoskeletal rearrangements induce a conformational change in the cell surface $\alpha_{\mathrm{v}} \beta_{6}$ integrin, which binds latent TGF- $\beta$ present in the extracellular matrix. This conformational change in $\alpha_{\mathrm{v}} \beta_{6}$ releases active TGF- $\beta$ from its latent complex, allowing it to interact with its cell surface receptors on neighboring cells and promote fibrosis $(26,27)$. Here we found that activated (cleaved) PAR1 was upregulated in the lungs in our vascular leak-dependent model of pulmonary fibrosis, and that this upregulation was effectively blocked with dabigatran treatment. This effect of dabigatran treatment suggests that PAR1 activation in this model is highly dependent on thrombin, although it is likely that at least some of the observed PAR1 activation was mediated by thrombinindependent mechanisms (e.g., factor Xa). Similar to observations from other mouse models of lung injury, we found that $\alpha_{\mathrm{v}} \beta_{6}$ is upregulated at sites of injury in our vascular leak-dependent model. We also found that thrombin inhibition significantly diminished lung $\alpha_{\mathrm{v}} \beta_{6}$ expression and activation of the canonical TGF- $\beta$ signaling pathway. Lung fibrosis in this model was also completely blocked by antibody neutralization of $\alpha_{v} \beta_{6}$. In aggregate, these data support a critical role for a thrombin/PAR $1 / \alpha_{v} \beta_{6} /$ TGF- $\beta$ axis in promoting lung fibrosis in this vascular leak-dependent model.

In addition to thrombin signaling through PAR1 to trigger epithelial cell $\alpha_{\mathrm{v}} \beta_{6}$-dependent TGF- $\beta$ activation, thrombin may also promote the development of lung fibrosis in this model through other 
mechanisms. Notably, thrombin has been shown to induce numerous profibrotic behaviors directly in fibroblasts, including proliferation, survival, myofibroblast differentiation, synthesis of extracellular matrix proteins, and expression of other profibrotic cytokines (56-60). Many of these behaviors appear to be dependent on thrombin's ability to cleave and activate PAR1 expressed on fibroblasts, and many have been shown to be inhibited by dabigatran (61). Activation of PAR1 on fibroblasts may induce these profibrotic behaviors through the protein kinase $C$ (PKC) pathway $(56,62)$. Alternatively, fibroblasts also express the $\alpha_{\mathrm{v}} \beta_{1}, \alpha_{\mathrm{v}} \beta_{3}$ and $\alpha_{\mathrm{v}} \beta_{5}$ integrins (63-65), which may activate latent TGF- $\beta$ in a manner similar to the epithelial cell-restricted $\alpha_{\mathrm{v}} \beta_{6}$. Lastly, although we did not detect significant differences in BAL leukocytes or BAL total protein concentration with thrombin inhibition in this model, we did detect subtle decreases in BAL albumin and $\alpha-2$ macroglobulin levels, suggesting an attenuation of vascular leak by thrombin inhibition. This is not surprising, since thrombin itself is known to induce endothelial barrier disruption (66). Therefore, it is also possible the protective effects of dabigatran on the development of fibrosis in this model may be partially due to the attenuation of vascular leak itself.

In considering the differential effects of direct thrombin inhibition and vitamin $\mathrm{K}$ antagonism, it is important to note that warfarin did not appear to significantly protect against lung fibrosis despite its potential to decrease the amount of active thrombin in the lungs - directly by decreasing prothrombin synthesis and indirectly by limiting the upstream processes in the coagulation cascade that lead to the conversion of prothrombin to thrombin. However, while total amounts of tissue thrombin should be decreased with warfarin treatment, warfarin would not be expected to have any effect on the proteolytic activity of any thrombin that is present in the lungs. Consistent with this hypothesis, we found that warfarin treatment failed to attenuate the increase in cleaved (activated) PAR1 in the lungs in this vascular leak-dependent model. We also found that warfarin failed to inhibit the upregulation of $\alpha_{v} \beta_{6}$ and the activation of TGF- $\beta$ signaling. Taken together, these data suggest that in the setting of therapeutic anticoagulation with warfarin, the residual levels of active thrombin are sufficient to activate the profibrotic PAR $1 / \alpha_{\mathrm{v}} \beta_{6} / \mathrm{TGF}-\beta$ axis. In contrast, direct thrombin inhibition appears to robustly inhibit the activation of this pathway, which correlates with protection from fibrosis. Figure 7 depicts a schematic representation of the proposed mechanistic differences between vitamin $\mathrm{K}$ antagonism and direct thrombin inhibition with respect to extravascular coagulation and lung fibrosis.

Another potential explanation for the lack of antifibrotic effect seen with warfarin treatment may be its effects on endogenous anticoagulant proteins. While vitamin $\mathrm{K}$ antagonism impairs the synthesis of several members of the coagulation cascade, namely factors II (prothrombin), VII, IX and X, it also impairs the synthesis of proteins $C$ and S, 2 endogenous inhibitors of coagulation. Of particular interest is protein C, which, when activated, signals through the endothelial protein $C$ receptor (EPCR) and has been shown to decrease endothelial permeability in vitro (67). Activated protein C (APC) has been shown to be protective against both lung injury and fibrosis in murine models $(68,69)$. Therefore, it is possible that any potentially antifibrotic effects of warfarin treatment may be counterbalanced by its potentially detrimental effect of reducing protein $\mathrm{C}$ levels.

Direct assessment of in vivo coagulation - and the activity of specific coagulation proteinases - has been difficult. One of the most commonly used techniques for assessing the activity of the coagulation cascade in vivo is the D-dimer assay, but this assay measures the soluble breakdown products of fibrin and is more accurately a reflection of fibrin turnover. Here we used a Gd-based fibrin-specific probe (EP-2104R) together with UTE-MRI to directly visualize fibrin deposition in the lung. Using this technique, we directly showed the increased lung fibrin deposition in our vascular leak-dependent model and the attenuation of this increase with dabigatran treatment. These imaging studies have potential clinical applications, as molecular imaging with fibrin-based probes could be used to assess the extent of coagulation cascade activation in the lungs of patients with pulmonary fibrosis. Although fibrin itself may not be required for the development of fibrosis, its deposition could serve as a biomarker of ongoing lung injury, thereby providing an assessment of IPF disease activity. In addition, noninvasive assessment of lung fibrin deposition could help stratify IPF patients based on their likelihood of experiencing a favorable response to direct thrombin inhibition, allowing for a personalized approach to the use of direct thrombin inhibitors for this disease.

In summary, we found that the development of lung fibrosis in a vascular leak-dependent model is highly dependent on thrombin activity and the epithelium-specific integrin $\alpha_{v} \beta_{6}$. Direct thrombin inhibition with dabigatran in this model significantly inhibited thrombin's downstream signaling pathways, including cleavage and activation of its major receptor PAR1, induction of the epithelium-specific integrin $\alpha_{v} \beta_{6}$, and 
activation of TGF- $\beta$. Dabigatran also significantly attenuated the development of fibrosis, as did antibody blockade of $\alpha_{v} \beta_{6}$. However, lung fibrosis appeared to be uncoupled from hemostasis and lung fibrin deposition in this model, as we found that therapeutic anticoagulation with warfarin was unable to downregulate the PAR $1 / \alpha_{v} \beta_{6} /$ TGF- $\beta$ axis or significantly protect against fibrosis. This dissociation of the antifibrotic effects of thrombin from hemostasis has important clinical implications, given recent findings that warfarin treatment is not beneficial in IPF (32), and the clinical availability of direct thrombin inhibitors that our data suggest could benefit these patients.

\section{Methods}

Vascular leak-dependent model of lung fibrosis. Adult male C57BL/6 mice, 7-9 weeks old ( 25 g), purchased from the NCI-Frederick Animal Production Program, were used for all experiments. Mice were administered a single intratracheal dose of bleomycin (Fresenius Kabi USA) at $0.1 \mathrm{U} / \mathrm{kg}$ on day 0 and administered intraperitoneal FTY720 (Cayman Chemical) at $1 \mathrm{mg} / \mathrm{kg} 3$ times per week, starting on day 0 and continuing for the duration of the experiments, as previously described (31). Control mice were administered intratracheal PBS and intraperitoneal vehicle on the same schedule. For dabigatran experiments, mice were fed with chow containing dabigatran at $10 \mathrm{mg} / \mathrm{g}$ (Harlan) or control chow starting on day 0 and continued for the duration of the experiments. For warfarin experiments, warfarin (Sigma-Aldrich) at $1 \mathrm{mg} / 1$ or vehicle $(0.1 \% \mathrm{DMSO})$ was added to the drinking water starting 7 days prior to bleomycin administration to achieve a steady state of anticoagulation, and continued for the duration of the experiments. For anti- $\alpha_{v} \beta_{6}$ experiments, the anti- $\alpha_{\mathrm{v}} \beta_{6}$ blocking antibody (3G9), generated as previously described (70), or a mouse IgG1 control Ab (anti-human LFA-3; 1E6) was administered at $1 \mathrm{mg} / \mathrm{kg} 3$ times per week, starting on day 0 . Mice were sacrificed at the time points indicated for analyses of blood, BAL, and lung tissue.

Assessments of systemic anticoagulation. After 1 week of treatment with dabigatran or 2 weeks of treatment with warfarin, whole blood was obtained by cardiac puncture at the time of sacrifice. Sodium citrate was added to whole blood at a 1:9 citrate/blood ratio, and the samples were inverted gently and then centrifuged at 1,500 $\mathrm{g}$ for 15 minutes. Plasma dTT or prothrombin times (PTs) were then performed at the Massachusetts General Hospital Special Coagulation Laboratory (71). Each individual mouse international normalized ratio (INR) was obtained by the following equation: mouse INR $=\left(\mathrm{PT} /[\text { mean control PT] })^{1.17}\right.$.

$B A L$ and lung tissue collection. BAL was performed with 6 successive $0.5-\mathrm{ml}$ aliquots of PBS ( $3 \mathrm{ml}$ total). These BAL samples were centrifuged at $540 \mathrm{~g}, 4^{\circ} \mathrm{C}$ for 5 minutes. The BAL supernatants were stored at $-80^{\circ} \mathrm{C}$ for later analyses. BAL cell pellets were resuspended in PBS for cell-count determination and cytospin analysis. For lung tissue analyses (Western blotting, measurement of extravascular D-dimer content), the lung vasculature was flushed with PBS, and then the lungs were excised, flash frozen on dry ice, and homogenized in PBS with 1× protease/phosphatase inhibitor cocktail (Thermo Fisher Scientific). Lung lysate was prepared by adding an equal volume of $2 \times$ RIPA buffer (Cell Signaling Technology) to an aliquot of lung homogenate, vortexing, and then centrifuging at $1,500 \mathrm{~g}, 4^{\circ} \mathrm{C}$ for 15 minutes. The supernatant was collected and stored at $-80^{\circ} \mathrm{C}$ for later analyses.

Analyses of lung inflammation, vascular leak, and extravascular coagulation. BAL supernatant total protein concentrations were determined using a commercially available BCA Protein Assay Kit (Pierce/Thermo Fisher Scientific), performed according to the manufacturer's instructions. BAL albumin and $\alpha-2$ macroglobulin concentrations were measured via ELISA kits specific for mouse albumin (Bethyl Laboratories) and mouse $\alpha-2$ macroglobulin (Abcam), performed according to the manufacturers' instructions. BAL total cell counts were determined using an automated cell counter (Nexcelom) and differential cell counts were determined on preparations of cells centrifuged with a Cytospin 3 (Shandon/Thermo Fisher Scientific) and stained with Hema 3 stain (EMD Millipore). Lung extravascular D-dimer concentrations were determined using a commercially available ELISA kit (Diagnostica Stago/Thermo Fisher Scientific) on lung homogenate samples collected as described above.

Lung hydroxyproline measurement. Total lung hydroxyproline was determined as described previously (31). Briefly, lungs were homogenized in PBS and hydrolyzed overnight in $6 \mathrm{~N} \mathrm{HCl}$ at $120^{\circ} \mathrm{C}$. A $25-\mu 1$ aliquot was then desiccated, resuspended in $25 \mu 1 \mathrm{H}_{2} \mathrm{O}$, and added to $0.5 \mathrm{ml}$ of $1.4 \%$ chloramine $\mathrm{T}$ (SigmaAldrich), 10\% n-propanol, and $0.5 \mathrm{M}$ sodium acetate, $\mathrm{pH}$ 6.0. After 20 minutes of incubation at room temperature, $0.5 \mathrm{ml}$ of Erlich's solution ( $1 \mathrm{M} p$-dimethylaminobenzaldehyde in $70 \% n$-propanol, $20 \%$ perchloric acid) was added and a 15 -minute incubation at $65^{\circ} \mathrm{C}$ performed. Absorbance was measured at 550 $\mathrm{nm}$ and the amount of hydroxyproline was determined against a standard curve. 
MRI of lung fibrin deposition. The Gd-based, fibrin-specific MRI probe EP-2104R was synthesized as described previously (44). The imaging protocol involved (a) multislice $2 \mathrm{D}$ rapid acquisition with refocused echo (RARE) imaging to delineate anatomy, (b) a baseline 3D UTE sequence with respiratory gating, (c) a baseline 3D fast low angle shot (FLASH) angiography sequence, (d) bolus injection of $10 \mu \mathrm{mol} / \mathrm{kg}$ of EP-2104R, (e) the 3D FLASH sequence was repeated 5 times, and (f) the 3D UTE sequence was repeated 12 minutes after injection. Animals were anesthetized with isoflurane (1\%-2\%) and placed in a specially designed cradle with body temperature maintained at $37^{\circ} \mathrm{C}$. The femoral vein was catheterized for intravenous delivery of the contrast agent while the animal was positioned in the scanner. Imaging was performed at $4.7 \mathrm{~T}$ using a small-bore animal scanner (Bruker Biospec) with a custom-built volume coil. A pneumatic pillow was placed on the mouse to enable respiratory monitoring and image gating (SA Instruments). The RARE acquisition was acquired in both axial and coronal orientations with repetition time/echo time $(\mathrm{TR} / \mathrm{TE})=1,000 \mathrm{~ms} / 6.5 \mathrm{~ms}$; matrix $=192 \times 192,11 \times 1 \mathrm{~mm}$ slices; RARE factor 8 ; field of view $(\mathrm{FOV})=$ $3.28 \times 3.28 \mathrm{~cm}$ for in-plane resolution of $170 \times 170 \mu \mathrm{m}$; and acquisition time $=2.2$ minutes. The $3 \mathrm{D}$ UTE sequence used TR/TE/flip angle $(\mathrm{FA})=8 \mathrm{~ms} / 0.02 \mathrm{~ms} / 45^{\circ}$, matrix $=192 \times 192 \times 192, \mathrm{FOV}=4.8 \times 4.8$ $\times 4.8 \mathrm{~cm}$, for $250 \times 250 \times 250 \mu \mathrm{m}$ resolution, resulting in an acquisition time of $\sim 30$ minutes when gated. Each 3D FLASH sequence used TR/TE/FA $=15.3 \mathrm{~ms} / 1.54 \mathrm{~ms} / 40^{\circ}$, matrix $=192 \times 96 \times 96, \mathrm{FOV}=4.8 \times$ $2.4 \times 2.4 \mathrm{~cm}$, for $250 \times 250 \times 250 \mu \mathrm{m}$ resolution, resulting in an acquisition time of 2.3 minutes. After imaging was performed, the amount of probe in the lungs was determined by taking an aliquot of the lung tissue homogenate and analyzing it by inductively coupled plasma mass spectrometry (ICP-MS)for Gd. Lutetium was used as an internal standard and the measured result was compared to a standard curve.

$M R$ image analysis. Images were analyzed using the program Osirix (www.osirix-viewer.com). For each animal the anatomic axial RARE images were used to define regions of interest (ROIs) in the lungs and in the air outside the animal. Care was taken to avoid large vessels and airways in the ROIs. These ROIs were copied to the same location in the pre- and postprobe UTE images. Lung ROIs ranged from 0.15 to 0.80 $\mathrm{cm}^{2}$, and the air ROIs were about $0.1 \mathrm{~cm}^{2}$. For each slice, the signal intensity (SI) in the lung was obtained. The standard deviation (SD) of the SI in the air adjacent to the animal was used to estimate the noise. For each UTE image slice, pre- and postprobe signal-to-noise ratio (SNR) was calculated as $\mathrm{SNR}=\mathrm{SI}_{\text {lung }} / \mathrm{SD}_{\text {air }}$. The percentage increase in signal postprobe was calculated as: \% signal increase $=\left(\mathrm{SNR}_{\text {Post }}-\mathrm{SNR}_{\mathrm{Pre}}\right) /$ $\mathrm{SNR}_{\mathrm{Pre}} \times 100$. For each healthy control animal, the average percentage signal increase in the lung ROIs was used to determine a base signal enhancement threshold. This threshold was calculated as the mean percentage lung signal increase for the healthy controls +3 SDs. Then, for all the mice the fraction of pixels within each ROI that met or surpassed the signal enhancement threshold was calculated.

Histological analysis and immunohistochemistry. Lungs were inflated with $10 \%$ buffered formalin at 25 $\mathrm{cmH}_{2} 0$ for 15 minutes, excised, and fixed in $10 \%$ formalin for 72 hours, and then embedded in paraffin. Paraffin-embedded 5 - $\mu \mathrm{m}$ sections were stained with Masson's trichrome stain. For immunohistochemical analyses, deparaffinized lung sections were incubated first with a human/mouse chimeric monoclonal anti- $\alpha_{\mathrm{v}} \beta_{6}$ primary $\mathrm{Ab}$ (ch2A1), produced as previously described (72), and then with a biotinylated goat anti-human IgG secondary antibody, and stained with the Vectastain ABC Kit (Vector Labs). Images were obtained with a Nikon Eclipse ME600 microscope with a Nikon DXM 1200C digital camera and NIS Elements AR 2.30 software.

Western blotting. Whole-lung or cell lysates were separated by SDS-PAGE then transferred to polyvinylidene difluoride (PVDF) membranes using the NuPAGE electrophoresis and transfer systems (Life Technologies), under reducing (for total SMAD2, p-SMAD2, cPAR1 and GAPDH) or nonreducing (for $\alpha_{\mathrm{v}} \beta_{6}$ and $\beta$-actin) conditions. After blocking with $5 \%$ milk or $3 \%$ BSA, membranes were incubated with antibodies against total p-SMAD2 (Cell Signaling Technology, 3108), total SMAD2 (Cell Signaling Technology, 3122), ch2A1, or anti-cPAR1 (Cleaved-Ser ${ }^{42}$; Sigma-Aldrich, SAB4500823) primary antibodies followed by horseradish peroxidase-conjugated secondary antibodies (Cell Signaling Technology), and then visualized with Amersham ECL chemiluminescent substrate (GE Healthcare). Membranes were then stripped and similarly reprobed for GAPDH or $\beta$-actin as loading controls.

Assessment of the specificity of anti-cPAR1. Fibroblasts isolated from discarded normal human lung tissue were grown to $\sim 80 \%$ confluence, serum starved overnight, and then washed with PBS and incubated in either control (serum-free) media, media containing $1 \mathrm{U} / \mathrm{ml}$ human thrombin (Sigma-Aldrich), or media containing thrombin $+1 \mu \mathrm{g} / \mathrm{ml}$ dabigatran (Cayman Chemical). After a 10-minute incubation, cells were trypsinized, washed with PBS, and lysed in RIPA buffer (Sigma-Aldrich) containing $1 \times$ protease/phosphatase inhibitor (Thermo Fisher Scientific). Western blotting for cPAR1 and GAPDH was performed as outlined above. 
Statistics. Except where indicated in the figure legends, differences between groups were analyzed for statistical significance with 2-tailed Student's $t$ tests and Mann-Whitney tests for parametric and nonparametric data, respectively, using Prism 6 software (GraphPad). $P$ values of less than 0.05 were considered statistically significant.

Study approval. All animal studies were approved by the Massachusetts General Hospital Subcommittee on Research Animal Care, and all mice were maintained in a specific pathogen-free environment certified by the American Association for Accreditation of Laboratory Animal Care (AAALAC).

\section{Author contributions}

BSS and AMT conceived of the overall project, designed the studies, and wrote the manuscript. PC and DES designed and directed the MRI studies. EMV designed and directed the analyses of mouse plasma coagulation parameters. PHW and SMV designed and directed the analyses of $\alpha_{v} \beta_{6}$ expression and produced, purified, and characterized the $3 \mathrm{G} 9$, ch2A1, and $1 \mathrm{E} 6$ antibodies. KEB provided purified human lung fibroblasts. CKP, PLB, NJR, and FB conducted the experiments and acquired data. All authors assisted with data analysis. All authors reviewed the manuscript and contributed to its intellectual content.

\section{Acknowledgments}

We gratefully acknowledge those agencies that funded this work, including grant numbers HL105656 (to BSS), HL108975 (to AMT), HL133153 (to AMT), HL109448 (to PC) and HL116315 (to PC), from the National Heart Lung and Blood Institute, EB009062 (to PC) from the National Institute of Biomedical Imaging and Bioengineering, and a sponsored research agreement from Biogen, Inc. (to AMT). ICP-MS instrumentation was funded by the NIH Office of the Director (OD010650).

Address correspondence to: Barry S. Shea, Division of Pulmonary, Critical Care and Sleep Medicine, Rhode Island Hospital, 593 Eddy Street, APC 7, Providence, Rhode Island 02903, USA. Phone: 401.444.6854; E-mail: barry_shea@brown.edu.

PLB's present address is: Division of Pulmonary and Critical Care Medicine, Feinberg School of Medicine, Northwestern University, Chicago, Illinois, USA.

FB's present address is: Department of Molecular Biotechnology and Health Sciences, University of Turin, Italy.

1. Selman M, King TE, Pardo A, American Thoracic Society, European Respiratory Society, American College of Chest Physicians. Idiopathic pulmonary fibrosis: prevailing and evolving hypotheses about its pathogenesis and implications for therapy. Ann Intern Med. 2001;134(2):136-151.

2. King TE, Pardo A, Selman M. Idiopathic pulmonary fibrosis. Lancet. 2011;378(9807):1949-1961.

3. Ahluwalia N, Shea BS, Tager AM. New therapeutic targets in idiopathic pulmonary fibrosis. Aiming to rein in runaway woundhealing responses. Am J Respir Crit Care Med. 2014;190(8):867-878.

4. Richeldi L, et al. Efficacy and safety of nintedanib in idiopathic pulmonary fibrosis. N Engl J Med. 2014;370(22):2071-2082.

5. King TE, et al. A phase 3 trial of pirfenidone in patients with idiopathic pulmonary fibrosis. NEngl J Med. 2014;370(22):2083-2092.

6. Selman M, Pardo A. Role of epithelial cells in idiopathic pulmonary fibrosis: from innocent targets to serial killers. Proc Am Thorac Soc. 2006;3(4):364-372.

7. Coward WR, Saini G, Jenkins G. The pathogenesis of idiopathic pulmonary fibrosis. Ther Adv Respir Dis. 2010;4(6):367-388.

8. Wynn TA, Ramalingam TR. Mechanisms of fibrosis: therapeutic translation for fibrotic disease. Nat Med. 2012;18(7):1028-1040.

9. Rinderknecht $\mathrm{J}$, et al. Accelerated clearance of small solutes from the lungs in interstitial lung disease. Am Rev Respir Dis. 1980;121(1):105-117.

10. Pantin CF, et al. Measures of the inflammatory response in cryptogenic fibrosing alveolitis. Am Rev Respir Dis. 1988;138(5):1234-1241.

11. Mogulkoc N, et al. Pulmonary (99m)Tc-DTPA aerosol clearance and survival in usual interstitial pneumonia (UIP). Thorax. 2001;56(12):916-923.

12. McKeown S, Richter AG, O'Kane C, McAuley DF, Thickett DR. MMP expression and abnormal lung permeability are important determinants of outcome in IPF. Eur Respir J. 2009;33(1):77-84

13. Chapman HA, Allen CL, Stone OL. Abnormalities in pathways of alveolar fibrin turnover among patients with interstitial lung disease. Am Rev Respir Dis. 1986;133(3):437-443.

14. Kotani I, Sato A, Hayakawa H, Urano T, Takada Y, Takada A. Increased procoagulant and antifibrinolytic activities in the lungs with idiopathic pulmonary fibrosis. Thromb Res. 1995;77(6):493-504.

15. Piguet PF, Van GY, Guo J. Heparin attenuates bleomycin but not silica-induced pulmonary fibrosis in mice: possible relationship 
with involvement of myofibroblasts in bleomycin, and fibroblasts in silica-induced fibrosis. Int J Exp Pathol. 1996;77(4):155-161.

16. Eitzman DT, et al. Bleomycin-induced pulmonary fibrosis in transgenic mice that either lack or overexpress the murine plasminogen activator inhibitor-1 gene. J Clin Invest. 1996;97(1):232-237.

17. Howell DC, et al. Direct thrombin inhibition reduces lung collagen, accumulation, and connective tissue growth factor mRNA levels in bleomycin-induced pulmonary fibrosis. Am J Pathol. 2001;159(4):1383-1395.

18. Günther A, et al. Prevention of bleomycin-induced lung fibrosis by aerosolization of heparin or urokinase in rabbits. Am J Respir Crit Care Med. 2003;168(11):1358-1365.

19. Scotton CJ, et al. Increased local expression of coagulation factor X contributes to the fibrotic response in human and murine lung injury. J Clin Invest. 2009;119(9):2550-2563.

20. Hattori N, et al. Bleomycin-induced pulmonary fibrosis in fibrinogen-null mice. J Clin Invest. 2000;106(11):1341-1350.

21. Ploplis VA, et al. A total fibrinogen deficiency is compatible with the development of pulmonary fibrosis in mice. Am J Pathol. 2000;157(3):703-708.

22. Howell DC, et al. Absence of proteinase-activated receptor-1 signaling affords protection from bleomycin-induced lung inflammation and fibrosis. Am J Pathol. 2005;166(5):1353-1365.

23. Bogatkevich GS, Ludwicka-Bradley A, Nietert PJ, Akter T, van Ryn J, Silver RM. Antiinflammatory and antifibrotic effects of the oral direct thrombin inhibitor dabigatran etexilate in a murine model of interstitial lung disease. Arthritis Rheum. 2011;63(5):1416-1425.

24. Lin C, et al. Targeting protease activated receptor-1 with P1pal-12 limits bleomycin-induced pulmonary fibrosis. Thorax. 2014;69(2):152-160.

25. Vu TK, Hung DT, Wheaton VI, Coughlin SR. Molecular cloning of a functional thrombin receptor reveals a novel proteolytic mechanism of receptor activation. Cell. 1991;64(6):1057-1068.

26. Jenkins RG, et al. Ligation of protease-activated receptor 1 enhances alpha(v)beta6 integrin-dependent TGF-beta activation and promotes acute lung injury. J Clin Invest. 2006;116(6):1606-1614.

27. Munger JS, et al. The integrin alpha $\mathrm{v}$ beta 6 binds and activates latent TGF beta 1: a mechanism for regulating pulmonary inflammation and fibrosis. Cell. 1999;96(3):319-328.

28. Horan GS, et al. Partial inhibition of integrin alpha(v)beta6 prevents pulmonary fibrosis without exacerbating inflammation Am J Respir Crit Care Med. 2008;177(1):56-65.

29. Puthawala K, et al. Inhibition of integrin alpha(v)beta6, an activator of latent transforming growth factor-beta, prevents radiation-induced lung fibrosis. Am J Respir Crit Care Med. 2008;177(1):82-90.

30. Saini G, et al. $\alpha v \beta 6$ integrin may be a potential prognostic biomarker in interstitial lung disease. Eur Respir J. 2015;46(2):486-494.

31. Shea BS, Brooks SF, Fontaine BA, Chun J, Luster AD, Tager AM. Prolonged exposure to sphingosine 1-phosphate receptor-1 agonists exacerbates vascular leak, fibrosis, and mortality after lung injury. Am J Respir Cell Mol Biol. 2010;43(6):662-673.

32. Noth I, et al. A placebo-controlled randomized trial of warfarin in idiopathic pulmonary fibrosis. Am J Respir Crit Care Med. 2012;186(1):88-95.

33. Duplantier JG, et al. A role for thrombin in liver fibrosis. Gut. 2004;53(11):1682-1687.

34. Fiorucci S, et al. PAR1 antagonism protects against experimental liver fibrosis. Role of proteinase receptors in stellate cell activation. Hepatology. 2004;39(2):365-375.

35. Sullivan BP, Weinreb PH, Violette SM, Luyendyk JP. The coagulation system contributes to alphaVbeta6 integrin expression and liver fibrosis induced by cholestasis. Am J Pathol. 2010;177(6):2837-2849.

36. Martinelli A, et al. Effect of a thrombin receptor (protease-activated receptor 1, PAR-1) gene polymorphism in chronic hepatitis C liver fibrosis. J Gastroenterol Hepatol. 2008;23(9):1403-1409.

37. Hewitson TD, Martic M, Kelynack KJ, Pagel CN, Mackie EJ, Becker GJ. Thrombin is a pro-fibrotic factor for rat renal fibroblasts in vitro. Nephron Exp Nephrol. 2005;101(2):e42-e49.

38. Hahm K, et al. Alphav beta6 integrin regulates renal fibrosis and inflammation in Alport mouse. Am J Pathol. 2007;170(1):110-125

39. Hawes EM, et al. Performance of coagulation tests in patients on therapeutic doses of dabigatran: a cross-sectional pharmacodynamic study based on peak and trough plasma levels. J Thromb Haemost. 2013;11(8):1493-1502.

40. Božič-Mijovski M, et al. Diluted thrombin time reliably measures low to intermediate plasma dabigatran concentrations. Ann Clin Biochem. 2016;53(Pt 4):446-451.

41. Johnson KM, Fain SB, Schiebler ML, Nagle S. Optimized 3D ultrashort echo time pulmonary MRI. Magn Reson Med. 2013;70(5):1241-1250.

42. Caravan P, et al. Molecular magnetic resonance imaging of pulmonary fibrosis in mice. Am J Respir Cell Mol Biol. 2013;49(6):1120-1126

43. Togao O, Tsuji R, Ohno Y, Dimitrov I, Takahashi M. Ultrashort echo time (UTE) MRI of the lung: assessment of tissue density in the lung parenchyma. Magn Reson Med. 2010;64(5):1491-1498.

44. Overoye-Chan K, et al. EP-2104R: a fibrin-specific gadolinium-Based MRI contrast agent for detection of thrombus. $J$ Am Chem Soc. 2008;130(18):6025-6039.

45. Botnar RM, et al. In vivo magnetic resonance imaging of coronary thrombosis using a fibrin-binding molecular magnetic resonance contrast agent. Circulation. 2004;110(11):1463-1466.

46. Spuentrup E, et al. Molecular magnetic resonance imaging of coronary thrombosis and pulmonary emboli with a novel fibrintargeted contrast agent. Circulation. 2005;111(11):1377-1382.

47. Spuentrup E, et al. Molecular magnetic resonance imaging of pulmonary emboli with a fibrin-specific contrast agent. Am $J$ Respir Crit Care Med. 2005;172(4):494-500.

48. Stracke CP, Katoh M, Wiethoff AJ, Parsons EC, Spangenberg P, Spüntrup E. Molecular MRI of cerebral venous sinus thrombosis using a new fibrin-specific MR contrast agent. Stroke. 2007;38(5):1476-1481.

49. Vymazal J, et al. Thrombus imaging with fibrin-specific gadolinium-based MR contrast agent EP-2104R: results of a phase II clinical study of feasibility. Invest Radiol. 2009;44(11):697-704.

50. Sheppard D. Integrin-mediated activation of transforming growth factor-beta(1) in pulmonary fibrosis. Chest. 2001;120(1 Suppl):49S-53S 
51. Wynn TA. Cellular and molecular mechanisms of fibrosis. J Pathol. 2008;214(2):199-210.

52. Bendstrup E, Hilberg O. Is warfarin the right anticoagulant in idiopathic pulmonary fibrosis? Am J Respir Crit Care Med. 2012;186(7):693; author reply 693-693; author reply 694

53. Bogatkevich GS, Nietert PJ, Silver RM, Highland KB. Rationale for anticoagulant therapy of pulmonary fibrosis. Am J Respir Crit Care Med. 2014;189(3):362-363.

54. Crooks MG, Hart SP. Coagulation and anticoagulation in idiopathic pulmonary fibrosis. Eur Respir Rev. 2015;24(137):392-399.

55. Schuepbach RA, Riewald M. Coagulation factor Xa cleaves protease-activated receptor-1 and mediates signaling dependent on binding to the endothelial protein C receptor. J Thromb Haemost. 2010;8(2):379-388.

56. Bogatkevich GS, Tourkina E, Silver RM, Ludwicka-Bradley A. Thrombin differentiates normal lung fibroblasts to a myofibroblast phenotype via the proteolytically activated receptor-1 and a protein kinase C-dependent pathway. J Biol Chem. 2001;276(48):45184-45192.

57. Ohba T, McDonald JK, Silver RM, Strange C, LeRoy EC, Ludwicka A. Scleroderma bronchoalveolar lavage fluid contains thrombin, a mediator of human lung fibroblast proliferation via induction of platelet-derived growth factor alpha-receptor. $A m J$ Respir Cell Mol Biol. 1994;10(4):405-412.

58. Chambers RC, Leoni P, Blanc-Brude OP, Wembridge DE, Laurent GJ. Thrombin is a potent inducer of connective tissue growth factor production via proteolytic activation of protease-activated receptor-1. J Biol Chem. 2000;275(45):35584-35591

59. Bogatkevich GS, et al. Distinct PKC isoforms mediate cell survival and DNA synthesis in thrombin-induced myofibroblasts. Am J Physiol Lung Cell Mol Physiol. 2005;288(1):L190-L201.

60. Chambers RC, Dabbagh K, McAnulty RJ, Gray AJ, Blanc-Brude OP, Laurent GJ. Thrombin stimulates fibroblast procollagen production via proteolytic activation of protease-activated receptor 1. Biochem J. 1998;333( Pt 1):121-127.

61. Bogatkevich GS, Ludwicka-Bradley A, Silver RM. Dabigatran, a direct thrombin inhibitor, demonstrates antifibrotic effects on lung fibroblasts. Arthritis Rheum. 2009;60(11):3455-3464.

62. Bogatkevich GS, Tourkina E, Abrams CS, Harley RA, Silver RM, Ludwicka-Bradley A. Contractile activity and smooth muscle alpha-actin organization in thrombin-induced human lung myofibroblasts. Am J Physiol Lung Cell Mol Physiol. 2003;285(2):L334-L343 .

63. Asano Y, Ihn H, Yamane K, Jinnin M, Mimura Y, Tamaki K. Increased expression of integrin alpha(v)beta3 contributes to the establishment of autocrine TGF-beta signaling in scleroderma fibroblasts. J Immunol. 2005;175(11):7708-7718.

64. Asano Y, Ihn H, Jinnin M, Mimura Y, Tamaki K. Involvement of alphavbeta5 integrin in the establishment of autocrine TGFbeta signaling in dermal fibroblasts derived from localized scleroderma. J Invest Dermatol. 2006;126(8):1761-1769.

65. Reed NI, et al. The $\alpha v \beta 1$ integrin plays a critical in vivo role in tissue fibrosis. Sci Transl Med. 2015;7(288):288ra79.

66. Gavard J, Gutkind JS. Protein kinase C-related kinase and ROCK are required for thrombin-induced endothelial cell permeability downstream from Galpha12/13 and Galpha11/q. J Biol Chem. 2008;283(44):29888-29896.

67. Finigan JH, et al. Activated protein C mediates novel lung endothelial barrier enhancement: role of sphingosine 1-phosphate receptor transactivation. J Biol Chem. 2005;280(17):17286-17293.

68. Finigan JH, et al. Activated protein C protects against ventilator-induced pulmonary capillary leak. Am J Physiol Lung Cell Mol Physiol. 2009;296(6):L1002-L1011.

69. Yasui $\mathrm{H}$, et al. Intratracheal administration of activated protein $\mathrm{C}$ inhibits bleomycin-induced lung fibrosis in the mouse. $A m \mathrm{~J}$ Respir Crit Care Med. 2001;163(7):1660-1668

70. Weinreb $\mathrm{PH}$, et al. Function-blocking integrin alphavbeta6 monoclonal antibodies: distinct ligand-mimetic and nonligandmimetic classes. J Biol Chem. 2004;279(17):17875-17887.

71. Kim YA, Gosselin R, Van Cott EM. The effects of dabigatran on lupus anticoagulant, diluted plasma thrombin time, and other specialized coagulation assays. Int J Lab Hematol. 2015;37(4):e81-e84

72. Van Aarsen LA, et al. Antibody-mediated blockade of integrin alpha v beta 6 inhibits tumor progression in vivo by a transforming growth factor-beta-regulated mechanism. Cancer Res. 2008;68(2):561-570. 